\title{
Face rings of simplicial complexes with singularities
}

\author{
Ezra Miller* \\ Mathematics Department, Box 90320 \\ Duke University, Durham, NC 27707, USA \\ ezra@math.duke.edu \\ Isabella Novik ${ }^{\dagger}$ \\ Department of Mathematics, Box 354350 \\ University of Washington, Seattle, WA 98195-4350, USA \\ novik@math. washington. edu \\ Ed Swartz $\ddagger$ \\ Department of Mathematics, \\ Cornell University, Ithaca NY, 14853-4201, USA \\ ebs22@cornell.edu
}

15 January 2010

\begin{abstract}
The face ring of a simplicial complex modulo $m$ generic linear forms is shown to have finite local cohomology if and only if the link of every face of dimension $m$ or more is nonsingular, i.e., has the homology of a wedge of spheres of the expected dimension. This is derived from an enumerative result for local cohomology of face rings modulo generic linear forms, as compared with local cohomology of the face ring itself. The enumerative result is generalized in slightly weaker form to squarefree modules. A concept of Cohen-Macaulay in codimension $c$ is defined and characterized for arbitrary finitely generated modules and coherent sheaves. For the face ring of an $r$-dimensional complex $\Delta$, it is equivalent to nonsingularity of $\Delta$ in dimension $r-c$; for a coherent sheaf on projective space, this condition is shown to be equivalent to the same condition on any single generic hyperplane section. The characterization of nonsingularity in dimension $m$ via finite local cohomology thus generalizes from face rings to arbitrary graded modules.
\end{abstract}

2010 Mathematics Subject Classification. 13F55, 05E40, 05E45, 13H10, 14M05, 13D45, 13C14

Keywords: simplicial complex, Stanley-Reisner (face) ring, Cohen-Macaulay, local cohomology, graded module, hyperplane section, squarefree module

\footnotetext{
${ }^{*}$ Research partially supported by NSF CAREER grant DMS-0449102

${ }^{\dagger}$ Research partially supported by Alfred P. Sloan Research Fellowship and NSF grant DMS-0801152

${ }_{\ddagger}^{\ddagger}$ Research partially supported by NSF grant DMS-0900912
} 


\section{Introduction}

In the 1970's Reisner (building on unpublished work of Hochster) and Stanley revolutionized the study of face enumeration of simplicial complexes through their use of the face ring, also called the Stanley-Reisner ring. Reisner proved that the face ring of a simplicial complex is Cohen-Macaulay if and only if the link of every face in the simplicial complex, including the empty face, is nonsingular, by which we mean that all reduced cohomology groups, except possibly in the maximum dimension, vanish [11]. Stanley used this to completely characterize the $f$-vectors of Cohen-Macaulay complexes [15].

A natural question which follows these results is, "What happens if singularities are allowed?" The weakest relaxation possible is to permit nontrivial cohomology of the whole complex (the link of the empty face) in lower dimensions. Schenzel proved that for pure complexes, the face ring is Buchsbaum if and only if this is the only additional cohomology of links [13]. The primary tool used in the proof of both Reisner's and Schenzel's theorems is local cohomology of the face ring with respect to the irrelevant ideal. In the CohenMacaulay case all but the top local cohomology modules vanish, while in the Buchsbaum case these modules have finite dimension as vector spaces. Rings with this property, that is, those whose local cohomology modules below their Krull dimension have finite dimension, are called generalized Cohen-Macaulay rings or rings with finite local cohomology.

Our first goal is to extend these ideas to arbitrary singularities. The main result along simplicial lines, Theorem [2.3, says that if the dimension of the singular set is $m-1$, then except for top cohomological degree, all of the local cohomology modules of the face ring modulo $m$ generic linear forms have finite dimension and vanish outside of $\mathbb{Z}$-graded degrees $i$ for $0 \leq i \leq m$. Theorem 2.3 is verified easily after Theorem 5.3. Theorem 5.3 is an enumerative result for Hilbert series of local cohomology of face rings modulo generic linear forms proved using a combinatorial description of the finely graded module structure of the local cohomology $H_{\mathfrak{m}}^{i}(\mathbb{k}[\Delta])$ due to Gräbe [6], which we review in Section 3. We treat isolated singularities in Section 4, and the general case in Section 5 .

Theorem 2.3 is not limited to a simplicial phenomenon; it is an instance of a rather general phenomenon in commutative algebra and algebraic geometry, stated and proved here in the language of sheaves on projective schemes as Theorem 7.7, and reinterpreted in terms of commutative algebra in Theorem 7.9. The first observation, made precise in Theorem 6.9, is that singularity in a fixed dimension is equivalent to a condition that we call Cohen-Macaulay in a fixed codimension: roughly speaking, the local rings at all points of some fixed codimension are Cohen-Macaulay. At first sight, this condition sounds like Serre's condition $S_{k}$, which has been treated in combinatorial settings such as affine semigroup rings and toric varieties [12, but it is subtly different; see Remark 6.10. The second, harder and deeper homological observation is that the Cohen-Macaulay condition in a fixed codimension satisfies a Bertini-type persistence under generic hyperplane section, but also the converse: if a single generic hyperplane section of a coherent sheaf is Cohen-Macaulay in codimension $c$, then so is the original sheaf; this is made precise in Theorem 7.7.

Having seen in Section 7 that Theorem 2.3 extends beyond the simplicial realm, we do the same for the enumerative result from which it follows: in Theorem 8.5 , we extend 
Theorem 5.3 to the squarefree modules introduced by Yanagawa [19].

\section{Singularity dimension and finite local cohomology}

For all undefined terminology we refer our readers to [1, 9, 16]. Throughout, $\Delta$ is a simplicial complex of dimension $d-1$ with vertex set $[n]=\{1, \ldots, n\}$, and $\mathbb{k}$ is a field. If $F \in \Delta$ is a face, then the link of $F$ is

$$
\operatorname{lk} F=\{G \in \Delta: F \cap G=\varnothing, F \cup G \in \Delta\} .
$$

In particular, $\mathrm{lk} \varnothing=\Delta$.

Definition 2.1. The face $F$ is nonsingular with respect to $\mathbb{k}$ if the reduced cohomology $\tilde{H}^{i}(\mathrm{lk} F ; \mathbb{k})$ of the link of $F$ with coefficients in $\mathbb{k}$ vanishes for all $i<d-1-|F|$. Otherwise $F$ is a singular face. The singularity dimension of $\Delta$ is the maximum dimension of a singular face. If $\Delta$ has no singular faces, then $\Delta$ is Cohen-Macaulay (over $\mathbb{k}$ ) and we (arbitrarily) declare the singularity dimension of the complex to be $-\infty$.

For a field $\mathbb{k}$, which we assume is infinite but of arbitrary characteristic, the face ring of $\Delta$ (also known as the Stanley-Reisner ring) is

$$
\mathbb{k}[\Delta]=\mathbb{k}\left[x_{1}, \ldots, x_{n}\right] / I_{\Delta},
$$

where the Stanley-Reisner ideal is

$$
I_{\Delta}=\left(x_{i_{1}} \cdots x_{i_{k}}:\left\{i_{1}, \ldots, i_{k}\right\} \notin \Delta\right) .
$$

For any module $M$ over the polynomial ring $S=\mathbb{k}\left[x_{1}, \ldots, x_{n}\right]$, we use $H_{\mathfrak{m}}^{i}(M)$ to denote the $i$-th local cohomology module of $M$ with respect to the irrelevant ideal $\mathfrak{m}=\left(x_{1}, \ldots, x_{n}\right)$.

Definition 2.2. Let $M$ be an $S$-module of Krull dimension $d$. Then $M$ is a module with finite local cohomology (or a generalized Cohen-Macaulay module) if $H_{\mathfrak{m}}^{i}(M)$ has finite dimension as a $\mathbb{k}$-vector space for all $i<d$.

Modules with finite local cohomology were introduced in [2], [17], and [18. Connections between face rings and modules with finite local cohomology have been studied in [5] and [20].

Here we show that the connection between algebraic properties of $\mathbb{k}[\Delta]$ and the singularities of the complex is given by the following.

Theorem 2.3. The singularity dimension of a simplicial complex $\Delta$ is less than $m$ if and only if for all sets $\left\{\theta_{1}, \ldots, \theta_{m}\right\}$ of $m$ generic linear forms the quotient $\mathbb{k}[\Delta] /\left(\theta_{1}, \ldots, \theta_{m}\right)$ is a ring with finite local cohomology.

We give two proofs: the one in Section 5 uses simplicial techniques on local cohomology; the one in Section 7 frames it as a special case of the general theory of modules and sheaves that are Cohen-Macaulay in a fixed codimension, via Theorem 6.9. 


\section{Local cohomology of face rings}

This section explains Gräbe's results on the combinatorial structure of the local cohomology of a face ring as a $\mathbb{Z}^{n}$-graded module over the polynomial ring $S=\mathbb{k}\left[x_{1}, \ldots, x_{n}\right]$.

Denote by $|\Delta|$ the geometric realization of $\Delta$. For a face $\tau \in \Delta$, the subcomplex

$$
\operatorname{cost} \tau=\{\sigma \in \Delta: \sigma \not \supset \tau\}
$$

is the contrastar of $\tau$. The $i$-th local cohomology of $\Delta$ along $\tau$ is the simplicial $i$-th cohomology

$$
H_{\tau}^{i}(\Delta):=H^{i}(\Delta, \operatorname{cost} \tau)
$$

of the pair $(\Delta$, cost $\tau)$ with coefficients in $\mathbb{k}$. For $\tau \subseteq \sigma \in \Delta$, the inclusion $\iota$ : cost $\tau \rightarrow \operatorname{cost} \sigma$ induces a contravariant map $\iota^{*}: H_{\sigma}^{i}(\Delta) \rightarrow H_{\tau}^{i}(\Delta)$.

For additional notation, an integer vector $U=\left(u_{1}, \ldots, u_{n}\right) \in \mathbb{Z}^{n}$ has support

$$
s(U)=\left\{\ell: u_{\ell} \neq 0\right\} \subseteq[n]
$$

and sum $|U|=\sum_{\ell=1}^{n} u_{\ell}$. Fix the standard basis $\left\{e_{\ell}\right\}_{\ell=1}^{n}$ for $\mathbb{Z}^{n}$, and write $\mathbb{N}$ for the set of nonnegative integers.

We consider the $\mathbb{Z}^{n}$-grading of $\mathbb{k}\left[x_{1}, \ldots, x_{n}\right]$ obtained by declaring the variable $x_{\ell}$ to have degree $e_{\ell}$. This grading refines the usual $\mathbb{Z}$-grading and induces a $\mathbb{Z}^{n}$-grading on $\mathbb{k}[\Delta]$ and its local cohomology modules; see [9. Chapter 13], for example. Thus, in particular, $H_{\mathfrak{m}}^{i}(\mathbb{k}[\Delta])_{j}=\bigoplus_{|U|=j} H_{\mathfrak{m}}^{i}(\mathbb{k}[\Delta])_{U}$, and multiplication by $x_{\ell}$ is a $\mathbb{k}$-linear map from $H_{\mathfrak{m}}^{i}(\mathbb{k}[\Delta])_{U}$ to $H_{\mathfrak{m}}^{i}(\mathbb{k}[\Delta])_{U+e_{\ell}}$ for all $U \in \mathbb{Z}^{n}$.

Theorem 3.1 (Gräbe). The following is an isomorphism of $\mathbb{Z}^{n}$-graded $\mathbb{k}[\Delta]$-modules:

$$
H_{\mathfrak{m}}^{i}(\mathbb{k}[\Delta]) \cong \bigoplus_{\substack{U \in \mathbb{N}^{n} \\ s(U) \in \Delta}} \mathcal{M}_{U}^{i}, \quad \text { where } \quad \mathcal{M}_{U}^{i}=H_{s(U)}^{i-1}(\Delta)
$$

and the $\mathbb{k}[\Delta]$-structure on the $U$-th component of the right-hand side is given by

$$
\cdot x_{\ell}= \begin{cases}0-\text { map } & \text { if } \ell \notin s(U) \\ \text { identity map } & \text { if } \ell \in s(U) \text { and } \ell \in s\left(U+e_{\ell}\right) \\ \iota^{*}: H_{s(U)}^{i-1}(\Delta) \rightarrow H_{s\left(U+e_{\ell}\right)}^{i-1}(\Delta) & \text { otherwise. }\end{cases}
$$

We note that the isomorphism of (11) on the level of vector spaces (rather than $\mathbb{k}[\Delta]$ modules) is attributed to Hochster in [16, Section II.4], and that

$$
H_{\mathfrak{m}}^{i}(\mathbb{k}[\Delta])_{0} \cong \mathcal{M}_{(0, \ldots, 0)}^{i}=H_{\varnothing}^{i-1}(\Delta)=\tilde{H}^{i-1}(\Delta ; \mathbb{k})
$$

is the reduced cohomology of $\Delta$ itself, by definition.

Corollary 3.2. Let $\Delta$ be a simplicial complex of dimension $d-1$. The singularity dimension of $\Delta$ is less than $m$ if and only if the standard $\mathbb{Z}$-graded Hilbert series $F\left(H_{\mathfrak{m}}^{i}(\mathbb{k}[\Delta]), 1 / \lambda\right)$ of $H_{\mathfrak{m}}^{i}(\mathbb{k}[\Delta])$ has a pole at $\lambda=1$ of order at most $m$ for $i<d$. 
Proof. An easy computation using eq. (11) implies that for $i<d$,

$F\left(H_{\mathfrak{m}}^{i}(\mathbb{k}[\Delta]), 1 / \lambda\right)=\sum_{F \in \Delta} \operatorname{dim}_{\mathbb{k}} H_{F}^{i-1}(\Delta) \cdot \frac{\lambda^{|F|}}{(1-\lambda)^{|F|}}=\sum_{F \in \Delta} \operatorname{dim}_{\mathbb{k}} \tilde{H}^{i-|F|-1}(\operatorname{lk} F ; \mathbb{k}) \cdot \frac{\lambda^{|F|}}{(1-\lambda)^{|F|}}$

(cf. [16, Theorem II.4.1]). The result now follows from the definition of singularity dimension.

\section{Simplicial isolated singularities}

Before proceeding to the proof of Theorem 2.3, we consider a special case.

Definition 4.1. $\Delta$ has isolated singularities if the singularity dimension of $\Delta$ is zero.

For the rest of this section we assume that $\Delta$ has isolated singularities. To begin with, we compute $H_{\mathfrak{m}}^{i}(\mathbb{k}[\Delta])$ for $i<d$. Since $\Delta$ has isolated singularities, Theorem 3.1 says that

$$
H_{\mathfrak{m}}^{i}(\mathbb{k}[\Delta])_{U}= \begin{cases}\tilde{H}^{i-1}(\Delta ; \mathbb{k}) & \text { if } U=0 \\ H_{\{t\}}^{i-1}(\Delta) & \text { if } s(U)=\{t\}, U \in-\mathbb{N}^{n} \\ 0 & \text { otherwise. }\end{cases}
$$

Let $\theta=\sum_{t=1}^{n} a_{t} x_{t}$ be a linear form in $S$ with $a_{t} \neq 0$ for all $t$. We use the exact sequence

$$
0 \rightarrow \mathbb{k}[\Delta] \stackrel{\cdot \theta}{\rightarrow} \mathbb{k}[\Delta] \rightarrow \mathbb{k}[\Delta] /(\theta) \rightarrow 0
$$

to compute $H_{\mathfrak{m}}^{i}(\mathbb{k}[\Delta] /(\theta))$ for $i<d-1$. (The Krull dimension of $\mathbb{k}[\Delta] /(\theta)$ is $d-1$.) Since the map $\cdot \theta$, that is multiplication by $\theta$, is injective for any face ring, the above sequence is in fact short exact. The corresponding long exact sequence in local cohomology is

$$
\cdots \rightarrow H_{\mathfrak{m}}^{i}(\mathbb{k}[\Delta])(-1) \stackrel{(\cdot \theta)^{*}}{\rightarrow} H_{\mathfrak{m}}^{i}(\mathbb{k}[\Delta]) \rightarrow H_{\mathfrak{m}}^{i}(\mathbb{k}[\Delta] /(\theta)) \stackrel{\delta}{\rightarrow} H_{\mathfrak{m}}^{i+1}(\mathbb{k}[\Delta])(-1) \stackrel{(\cdot \theta)^{*}}{\rightarrow} \ldots
$$

Here, $\delta$ is the connecting homomorphism, the notation $(-1)$ indicates a $\mathbb{Z}$-graded shift up by 1 (thus $M(-1)_{j}=M_{j-1}$ for any $\mathbb{Z}$-graded $S$-module $M$ ), and $(\cdot \theta)^{*}$ is the map induced by multiplication, hence it is just the module action of multiplication by $\theta$ on $H_{\mathfrak{m}}^{i}(\mathbb{k}[\Delta])$.

Let $f^{i}: \bigoplus_{t=1}^{n} H_{\{t\}}^{i}(\Delta) \rightarrow H_{\varnothing}^{i}(\Delta)$ be the map on simplicial local cohomology defined by

$$
f^{i}=\sum_{t=1}^{n} a_{t} \cdot \iota^{*}\left[H_{\{t\}}^{i}(\Delta) \rightarrow H_{\varnothing}^{i}(\Delta)\right] .
$$

By Gräbe's description of the $S$-module structure of the local cohomology modules, using the standard $\mathbb{Z}$-grading (in which $\operatorname{deg} x_{t}=1 \in \mathbb{Z}$ for all $t$ ) and the above long exact sequence,

$$
H_{\mathfrak{m}}^{i}(\mathbb{k}[\Delta] /(\theta))_{j} \cong\left\{\begin{array}{ll}
0 & \text { if } j<0 \\
\left(\operatorname{coker} f^{i-1}\right) \oplus\left(\operatorname{ker} f^{i}\right) & \text { if } j=0 \\
H_{\varnothing}^{i}(\Delta) & \text { if } j=1
\end{array} \quad(\forall i<d-1) .\right.
$$


In particular, $H_{\mathfrak{m}}^{i}(\mathbb{k}[\Delta] /(\theta))$ has finite dimension as a vector space over $\mathbb{k}$ for $i<d-1$.

Under certain conditions it is possible to extract even more precise information. We say that $\Delta$ has homologically isolated singularities if in dimensions $i$ in the range $0 \leq i \leq d-2$, the kernel of the above map $f^{i}$ decomposes as a direct sum:

$$
\operatorname{ker} f^{i}=\bigoplus_{t=1}^{n} \operatorname{ker} \iota^{*}\left[H_{\{t\}}^{i}(\Delta) \rightarrow H_{\varnothing}^{i}(\Delta)\right] \text {. }
$$

Equivalently, $\Delta$ has homologically isolated singularities if for $0 \leq i \leq d-2$, the images $\iota^{*}\left(H_{\{t\}}^{i}(\Delta)\right)$ for $t=1, \ldots, n$ are linearly independent subspaces of $H_{\varnothing}^{i}(\Delta)$. Evidently, any complex with only one isolated singularity has homologically isolated singularities. Other possibilities include (among many) pinching off homologically independent handles of a handlebody or coning off boundary components of a manifold-with-boundary having appropriate homological properties.

Suppose $\Delta$ has homologically isolated singularities. In addition to the vector space structure of (2), the $\mathbb{Z}$-grading implies that the $S$-module structure of $H_{\mathfrak{m}}^{i}(\mathbb{k}[\Delta] /(\theta))_{j}$ is trivial for $j=1$ and for $j=0$ restricted to coker $f^{i-1}$. Furthermore, on ker $f^{i} \subseteq H_{\mathfrak{m}}^{i}(\mathbb{k}[\Delta] /(\theta))_{0}$ the $S$-module structure is still induced by $\iota$, and hence also trivial. Therefore, by [17, Proposition I.3.10], $\mathbb{k}[\Delta] /(\theta)$ is Buchsbaum. Thus, the Hilbert function of $\mathbb{k}[\Delta] /\left(\theta_{1}, \ldots, \theta_{d}\right)$ for any linear system of parameters (l.s.o.p.) $\left\{\theta_{1}, \ldots, \theta_{d}\right\}$ is completely determined by the topology of $\Delta$ and its $f$-vector. (Indeed, since $\mathbb{k}$ is infinite, any l.s.o.p. for $\Delta$ will contain in its $\mathbb{k}$ linear span an element which is nonzero on every vertex and this one can be used as $\theta_{1}$.) This is a well known property of Cohen-Macaulay [11] and, more generally, Buchsbaum complexes [13. For an arbitrary complex $\Delta$, distinct linear systems of parameters can produce different Hilbert functions for the Artinian quotient $\mathbb{k}[\Delta] /\left(\theta_{1}, \ldots, \theta_{d}\right)$. However, for sufficiently generic linear systems of parameters, the Hilbert function of $\mathbb{k}[\Delta] /\left(\theta_{1}, \ldots, \theta_{d}\right)$ is constant. A natural question is the following.

Question 4.2. Let $\Delta_{1}$ and $\Delta_{2}$ be two simplicial complexes whose geometric realizations are homeomorphic and whose f-vectors are identical. Are the Hilbert functions of their Artinian quotients obtained from sufficiently generic linear systems of parameters the same?

\section{Main enumerative theorem for face rings}

We now proceed to the proof of the main theorem. As before, let $\Delta$ be a $(d-1)$-dimensional simplicial complex on $[n]$. Let $\theta_{1}, \ldots, \theta_{d}$ be $d$ generic linear forms in $S$ with $\theta_{p}=\sum_{t=1}^{n} a_{t, p} x_{t}$. In particular, we assume that every square submatrix of the $n \times d$-matrix $A=\left(a_{t, p}\right)$ is nonsingular and the $\theta$ 's satisfy the prime avoidance argument in the proof of Theorem 5.3 below.

Each $\theta_{p}$ acts on $\mathbb{k}[\Delta]$ by multiplication, $\cdot \theta_{p}: \mathbb{k}[\Delta] \rightarrow \mathbb{k}[\Delta]$. This map, in turn, induces the map $\left(\cdot \theta_{p}\right)^{*}=\cdot \theta_{p}: H_{\mathfrak{m}}^{\ell}(\mathbb{k}[\Delta]) \rightarrow H_{\mathfrak{m}}^{\ell}(\mathbb{k}[\Delta])$ that increases the $\mathbb{Z}$-grading by 1 . The key objects in the proof are the kernels of these maps and their intersections:

$$
\operatorname{ker}_{m, i}^{\ell}:=\bigcap_{p=1}^{m}\left(\operatorname{ker}\left(\cdot \theta_{p}\right)^{*}: H_{\mathfrak{m}}^{\ell}(\mathbb{k}[\Delta])_{-(i+1)} \rightarrow H_{\mathfrak{m}}^{\ell}(\mathbb{k}[\Delta])_{-i}\right) \quad \text { and } \quad \operatorname{ker}_{m}^{\ell}:=\bigoplus_{i \in \mathbb{Z}} \operatorname{ker}_{m, i}^{\ell} .
$$


Thus $\operatorname{ker}_{m}^{\ell}$ is a graded submodule of $H_{\mathfrak{m}}^{\ell}(\mathbb{k}[\Delta])$ and $\operatorname{ker}_{m, i}^{\ell}$ is simply $\left(\operatorname{ker}_{m}^{\ell}\right)_{-(i+1)}$.

What are the dimensions of these kernels? If $m=0$, then $\operatorname{ker}_{0, i}^{\ell}=H_{\mathfrak{m}}^{\ell}(\mathbb{k}[\Delta])_{-(i+1)}$, and Theorem 3.1 implies that for $i \geq 0$,

$$
\begin{aligned}
\operatorname{dim}_{\mathbb{k}} \operatorname{ker}_{0, i}^{\ell} & =\sum_{F \in \Delta}\left|\left\{U \in \mathbb{N}^{n}: s(U)=F,|U|=i+1\right\}\right| \cdot \operatorname{dim}_{\mathbb{k}} H_{F}^{\ell-1}(\Delta) \\
& =\sum_{F \in \Delta}\left(\begin{array}{c}
i \\
|F|-1
\end{array}\right) \cdot \operatorname{dim}_{\mathbb{k}} H_{F}^{\ell-1}(\Delta) .
\end{aligned}
$$

For a general $m$, we prove the following (where we set $\left(\begin{array}{l}a \\ b\end{array}\right)=0$ if $b<0$ ).

Lemma 5.1. For every $0 \leq m \leq d$, $\ell \leq d$, and $i \geq m$,

$$
\operatorname{dim}_{\mathbb{k}} \operatorname{ker}_{m, i}^{\ell} \leq \sum_{F \in \Delta}\left(\begin{array}{c}
i-m \\
|F|-m-1
\end{array}\right) \cdot \operatorname{dim}_{\mathbb{k}} H_{F}^{\ell-1}(\Delta) .
$$

We defer the proof to the end of the section. Using Lemma 5.1, we can say even more.

Lemma 5.2. For every $0 \leq m \leq d$, $\ell \leq d$, and $i \geq m$,

$$
\operatorname{dim}_{\mathbb{k}} \operatorname{ker}_{m, i}^{\ell}=\sum_{F \in \Delta}\left(\begin{array}{c}
i-m \\
|F|-m-1
\end{array}\right) \cdot \operatorname{dim}_{\mathbb{k}} H_{F}^{\ell-1}(\Delta) .
$$

Moreover, the map $\oplus_{i \geq m+1} \operatorname{ker}_{m, i}^{\ell} \stackrel{\cdot \theta_{m+1}}{\longrightarrow} \oplus_{i \geq m+1} \operatorname{ker}_{m, i-1}^{\ell}$, is a surjection.

Proof. We prove the statement on the dimension of $\operatorname{ker}_{m, i}^{\ell}$ by induction on $m$. For $m=0$ (and any $\ell, i \geq 0$ ), this is eq. (3). For larger $m$, we notice that the restriction of $\left(\cdot \theta_{m+1}\right)^{*}$ to $\operatorname{ker}_{m, i}^{\ell}$ is a linear map from $\operatorname{ker}_{m, i}^{\ell}$ to $\operatorname{ker}_{m, i-1}^{\ell}$, whose kernel is $\operatorname{ker}_{m+1, i}^{\ell}$. Thus for $i \geq m+1$,

$$
\begin{aligned}
\operatorname{dim}_{\mathbb{k}} \operatorname{ker}_{m+1, i}^{\ell} & \geq \operatorname{dim}_{\mathbb{k}} \operatorname{ker}_{m, i}^{\ell}-\operatorname{dim} \operatorname{ker}_{m, i-1}^{\ell} \\
& =\sum_{F \in \Delta}\left[\left(\begin{array}{c}
i-m \\
|F|-m-1
\end{array}\right)-\left(\begin{array}{c}
i-1-m \\
|F|-m-1
\end{array}\right)\right] \cdot \operatorname{dim}_{\mathbb{k}} H_{F}^{\ell-1}(\Delta) \\
& =\sum_{F \in \Delta}\left(\begin{array}{c}
i-(m+1) \\
|F|-(m+1)-1
\end{array}\right) \cdot \operatorname{dim}_{\mathbb{k}} H_{F}^{\ell-1}(\Delta) .
\end{aligned}
$$

The second step in the above computation is by the inductive hypothesis. Comparing the resulting inequality to that of Lemma 5.1 shows that this inequality is in fact equality, and hence that the map $\left(\cdot \theta_{m+1}\right)^{*}: \operatorname{ker}_{m, i}^{\ell} \rightarrow \operatorname{ker}_{m, i-1}^{\ell}$ is surjective for $i \geq m+1$.

Lemma 5.2 allows us to get a handle on $H_{\mathfrak{m}}^{\ell}\left(\mathbb{k}[\Delta] /\left(\theta_{1}, \ldots, \theta_{m}\right)\right)_{-i}$ at least for $\ell, i>0$.

Theorem 5.3. For $0 \leq m \leq d$ and $0<\ell \leq d-m$, there is a graded isomorphism of modules

$$
\bigoplus_{i \geq 1} H_{\mathfrak{m}}^{\ell}\left(\mathbb{k}[\Delta] /\left(\theta_{1}, \ldots, \theta_{m}\right)\right)_{-i} \cong \bigoplus_{i \geq 1} \operatorname{ker}_{m, i+m-1}^{\ell+m}
$$


compatible with the direct sum, hence $H_{\mathfrak{m}}^{\ell}\left(\mathbb{k}[\Delta] /\left(\theta_{1}, \ldots, \theta_{m}\right)\right)_{-i} \cong \operatorname{ker}_{m, i+m-1}^{\ell+m}$. Thus,

$$
\operatorname{dim}_{\mathbb{k}} H_{\mathfrak{m}}^{\ell}\left(\mathbb{k}[\Delta] /\left(\theta_{1}, \ldots, \theta_{m}\right)\right)_{-i}=\sum_{F \in \Delta}\left(\begin{array}{c}
i-1 \\
|F|-m-1
\end{array}\right) \cdot \operatorname{dim}_{\mathbb{k}} H_{F}^{\ell+m-1}(\Delta) \quad \text { for } \ell, i>0 .
$$

Proof. The proof is by induction on $m$, with the $m=0$ case being evident. For larger $m$, we want to mimic the proof given in Section 4. One obstacle to this approach is that the map $\cdot \theta_{m+1}: \mathbb{k}[\Delta] /\left(\theta_{1}, \ldots, \theta_{m}\right) \rightarrow \mathbb{k}[\Delta] /\left(\theta_{1}, \ldots, \theta_{m}\right)=: \mathcal{M}[m]$ might not be injective anymore. However, a "prime avoidance" argument together with the genericity assumption on $\theta_{m+1}$ implies that $\cdot \theta_{m+1}: \mathcal{M}[m] / H_{\mathfrak{m}}^{0}(\mathcal{M}[m]) \rightarrow \mathcal{M}[m] / H_{\mathfrak{m}}^{0}(\mathcal{M}[m])$ is injective, and hence one has the corresponding long exact sequence in local cohomology; see, for instance, [3, Chapter 3] for details on "prime avoidance" arguments. Since $H_{\mathfrak{m}}^{0}(\mathcal{M}[m])$ has Krull dimension 0, mod$\operatorname{ding} H_{\mathfrak{m}}^{0}(\mathcal{M}[m])$ out does not affect $H_{\mathfrak{m}}^{\ell}$ for $\ell>0$, so that the part of this long exact sequence corresponding to $\ell, i>0$ can be rewritten as

$$
\begin{aligned}
\bigoplus_{i \geq 1} H_{\mathfrak{m}}^{\ell}(\mathcal{M}[m])_{-(i+1)} & \stackrel{\left(\cdot \theta_{m+1}\right)^{*}}{\longrightarrow} \bigoplus_{i \geq 1} H_{\mathfrak{m}}^{\ell}(\mathcal{M}[m])_{-i} \longrightarrow \bigoplus_{i \geq 1} H_{\mathfrak{m}}^{\ell}(\mathcal{M}[m+1])_{-i} \\
& \stackrel{\delta}{\longrightarrow} \bigoplus_{i \geq 1} H_{\mathfrak{m}}^{\ell+1}(\mathcal{M}[m])_{-(i+1)} \stackrel{\left(\cdot \theta_{m+1}\right)^{*}}{\longrightarrow} \bigoplus_{i \geq 1} H_{\mathfrak{m}}^{\ell+1}(\mathcal{M}[m])_{-i} .
\end{aligned}
$$

By the inductive hypothesis combined with Lemma 5.2, the leftmost map in this sequence is surjective. Hence the module $\oplus_{i \geq 1} H_{\mathfrak{m}}^{\ell}(\mathcal{M}[m+1])_{-i}$ is isomorphic to the kernel of the rightmost map. Applying the inductive hypothesis to the last two entries of the sequence then implies the following isomorphism of modules:

$$
\bigoplus_{i \geq 1} H_{\mathfrak{m}}^{\ell}(\mathcal{M}[m+1])_{-i} \cong \bigoplus_{i \geq 1} \operatorname{ker}\left(\left(\cdot \theta_{m+1}\right)^{*}: \operatorname{ker}_{m, i+m}^{\ell+m+1} \rightarrow \operatorname{ker}_{m, i+m-1}^{\ell+m+1}\right)=\bigoplus_{i \geq 1} \operatorname{ker}_{m+1, i+m}^{\ell+m+1}
$$

Theorem 2.3 now follows easily from Theorem 5.3.

Proof of Theorem $\left[2.3\right.$. Since $\mathbb{k}[\Delta]$ is a finitely-generated algebra, $H_{\mathfrak{m}}^{0}\left(\mathbb{k}[\Delta] /\left(\theta_{1}, \ldots, \theta_{m}\right)\right)$ has Krull dimension zero, and hence is a finite-dimensional vector space for any simplicial complex $\Delta$. So we only need to care about $H_{\mathfrak{m}}^{\ell}$ for $\ell>0$. As $\left(\begin{array}{c}i-1 \\ |F|-m-1\end{array}\right)>0$ for all $i \gg 0$ and $|F|>m$, Theorem 5.3 implies that $\mathbb{k}[\Delta] /\left(\theta_{1}, \ldots, \theta_{m}\right)$ is a ring with finite local cohomology if and only if for all faces $F \in \Delta$ of size larger than $m$ and all $\ell+m<d$, the cohomology $H_{F}^{\ell+m-1}(\Delta)$ vanishes. Given that $H_{F}^{\ell+m-1}(\Delta)$ is isomorphic to $\tilde{H}^{\ell+m-1-|F|}(\mathrm{lk} F ; \mathbb{k}$ ) (see e.g. [6. Lemma 1.3]), this happens if and only if each such $F$ is nonsingular.

It remains to verify Lemma 5.1. For its proof we use the following notation. Fix $m, \ell>0$, and $i \geq m$. For $r \in\{i, i+1\}$, let

$$
\mathbf{V}_{r}:=\left\{U \in \mathbb{N}^{n}:|U|=r, s(U) \in \Delta\right\}
$$

and for $F \in \Delta$, let $\mathbf{V}_{r, F}:=\left\{u \in \mathbf{V}_{r}: s(U)=F\right\}$. If $F=\left\{f_{1}<\cdots<f_{j}\right\} \in \Delta$ where $j>m$, then set $\mathbf{W}_{r, F}:=\left\{U=\left(u_{1}, \ldots, u_{n}\right) \in \mathbf{V}_{r, F}: u_{f_{s}}=1\right.$ for $\left.1 \leq s \leq m\right\}$. Observe that $\mathbf{W}_{i+1, F}$ is a subset of $\mathbf{V}_{i+1, F}$ of cardinality $\left(\begin{array}{c}i-m \\ |F|-m-1\end{array}\right)$. 
For $G \subseteq F \in \Delta$ let $\Phi_{F, G}$ denote the map $\iota^{*}: H_{F}^{\ell-1}(\Delta) \rightarrow H_{G}^{\ell-1}(\Delta)$. Thus, $\Phi_{F, G}$ is the identity map if $F=G$. Using Theorem 3.1, we identify $H_{\mathfrak{m}}^{\ell}(\mathbb{k}[\Delta])_{-r}$ with $\bigoplus_{U \in \mathbf{V}_{r}} H_{s(U)}^{\ell-1}(\Delta)$, and for $z \in H_{\mathfrak{m}}^{\ell}(\mathbb{k}[\Delta])_{-r}$ we write $z=\left(z_{U}\right)_{U \in \mathbf{V}_{r}}$, where $z_{U} \in H_{s(U)}^{\ell-1}(\Delta)$ is the $(-U)$-th component of $z$. Since $\theta_{p}=\sum_{t=1}^{n} a_{t, p} x_{t}$, Theorem 3.1 yields that for such $z, r=i+1$, and $T \in \mathbf{V}_{i}$,

$$
\left(\theta_{p} z\right)_{T}=\sum_{\left\{t: T+e_{t} \in \mathbf{V}_{i+1}\right\}} a_{t, p} \cdot \Phi_{s\left(T+e_{t}\right), s(T)}\left(z_{T+e_{t}}\right) .
$$

Proof of Lemma 5.1. Since $\left|\mathbf{W}_{i+1, F}\right|=\left(\begin{array}{c}i-m \\ |F|-m-1\end{array}\right)$ for all $F \in \Delta$, to prove that $\operatorname{dim}_{\mathbb{k}} \operatorname{ker}_{m, i}^{\ell} \leq$ $\sum_{F \in \Delta}\left(\begin{array}{c}i-m \\ |F|-m-1\end{array}\right) \cdot \operatorname{dim}_{\mathbb{k}} H_{F}^{\ell-1}(\Delta)$, it is enough to verify that for $z, z^{\prime} \in \operatorname{ker}_{m, i}^{\ell}$,

$$
z_{U}=z_{U}^{\prime} \text { for all } F \in \Delta \text { and all } U \in \mathbf{W}_{i+1, F} \quad \Longrightarrow \quad z=z^{\prime}
$$

or equivalently (since $\operatorname{ker}_{m, i}^{\ell}$ is a $\mathbb{k}$-space) that for $z \in \operatorname{ker}_{m, i}^{\ell}$,

$$
z_{U}=0 \text { for all } F \in \Delta \text { and all } U \in \mathbf{W}_{i+1, F} \quad \Longrightarrow \quad z=0 \text {. }
$$

To prove this, fix such a $z$. From eq. (41) and the definition of $\operatorname{ker}_{m, i}^{\ell}$, it follows that

$$
\sum_{\left\{t: T+e_{t} \in \mathbf{V}_{i+1}\right\}} a_{t, p} \cdot \Phi_{s\left(T+e_{t}\right), s(T)}\left(z_{T+e_{t}}\right)=0 \quad \forall 1 \leq p \leq m \text { and } \forall T \in \mathbf{V}_{i} .
$$

For a given $T \in \mathbf{V}_{i}$, we refer to the $m$ conditions imposed on $z$ by eq. (6) as "the system defined by $T$ ", and denote this system by $\mathcal{S}_{T}$.

Define a partial order, $\succ$, on $\mathbf{V}_{i}$ as follows: $T^{\prime} \succ T$ if either $\left|s\left(T^{\prime}\right)\right|>|s(T)|$, or $s\left(T^{\prime}\right)=$ $s(T)$ and the last non-zero entry of $T^{\prime}-T$ is positive. To finish the proof, we verify by a descending (with respect to $\succ$ ) induction on $T \in \mathbf{V}_{i}$, that $z_{T+e_{t}}=0$ for all $t$ with $T+e_{t} \in$ $\mathbf{V}_{i+1}$. For $T \in \mathbf{V}_{i}$, there are two possible cases (we assume that $s(T)=F=\left\{f_{1}<\ldots<f_{j}\right\}$ ).

Case 1. $T \in \mathbf{W}_{i, F}$. Then for each $t \notin F$, either $F^{\prime}:=F \cup\{t\} \notin \Delta$, in which case $T+e_{t} \notin \mathbf{V}_{i+1}$, or $T+e_{t} \in \mathbf{W}_{i+1, F^{\prime}}$, in which case $z_{T+e_{t}}=0$ by eq. (5). Similarly, if $t=f_{r}$ for some $r>m$, then $T+e_{t} \in \mathbf{W}_{i+1, F}$, and $z_{T+e_{t}}=0$ by (15). Finally, for any $t \in F$, $s\left(T+e_{t}\right)=s(T)=F$, and so $\Phi_{s\left(T+e_{t}\right), s(T)}$ is the identity map. Thus the system $\mathcal{S}_{T}$ reduces to $m$ linear equations in $m$ variables:

$$
\sum_{r=1}^{m} a_{f_{r}, p} \cdot z_{T+e_{f_{r}}}=0 \quad \forall 1 \leq p \leq m
$$

Since the matrix $\left(a_{f_{r}, p}\right)_{1 \leq r, p \leq m}$ is nonsingular, $\left(z_{T+e_{t}}=0\right.$ for all $\left.t\right)$ is the only solution of $\mathcal{S}_{T}$.

Case 2. $T \notin \mathbf{W}_{i, F}$, and so $T_{f_{s}} \geq 2$ for some $s \leq m$. Then for any $t \notin F, T^{\prime}:=T-e_{f_{s}}+e_{t} \succ$ $T$, as $T^{\prime}$ has a larger support than $T$, and $T^{\prime}+e_{f_{s}}=T+e_{t}$. Hence $z_{T+e_{t}}=z_{T^{\prime}+e_{f_{s}}}=0$ by the inductive hypothesis on $T^{\prime}$. Similarly, if $t \in F-\left\{f_{1}, \ldots, f_{m}\right\}$, then $t>f_{s}$, and so $T^{\prime \prime}:=T-e_{f_{s}}+e_{t} \succ T$. As $T^{\prime \prime}+e_{f_{s}}=T+e_{t}$, the inductive hypothesis on $T^{\prime \prime}$ imply that $z_{T+e_{t}}=0$. Thus, as in Case $1, \mathcal{S}_{T}$ reduces to system (17), whose only solution is trivial. 


\section{Complexes Cohen-Macaulay in a fixed codimension}

Our goal for this section, in Theorem 6.9, is to rephrase the notion of singularity dimension of a simplicial complex $\Delta$ as a local geometric condition on the (spectrum of) the face ring $\mathbb{k}[\Delta]$, analogous to - and generalizing - the Cohen-Macaulay condition.

Definition 6.1. A simplicial complex $\Delta$ is $C M$ of dimension $i$ along $F$ if lk $F$ is CohenMacaulay of dimension $i$.

Remark 6.2. If $\Delta$ has dimension $d-1$, then for $\Delta$ to be CM of dimension $d-1-|F|$ along $F$ is stronger than for $F$ to be a nonsingular face of $\Delta$. For example, nonsingularity of $\varnothing$ means only that $\Delta$ is a homology-wedge-of-spheres, while $\mathrm{CM}$ of dimension $d-1$ along $\varnothing$ implies nonsingularity of all faces of $\Delta$.

Definition 6.3. A simplicial complex $\Delta$ of dimension $r$ is $C M$ in codimension $c$ if $\Delta$ is $C M$ of dimension $c-1$ along every face of dimension $r-c$, or if $c>r+1$ and $\Delta$ is Cohen-Macaulay.

Remark 6.4. If $\Delta$ is pure, then it would be enough to require that lk $F$ is Cohen-Macaulay for every face $F$ of dimension $r-c$; but if $\Delta$ has dimension $r$ and is not pure, then it is possible for lk $F$ to be Cohen-Macaulay without $\Delta$ being CM of dimension $r-|F|$ along $F$ (such a face $F$ is singular in $\Delta$ unless $l k F$ is $\mathbb{k}$-acyclic). This occurs, for example, when $F$ is a facet of dimension $<r$, in which case $l \mathrm{k} F=\{\varnothing\}$ is Cohen-Macaulay of dimension -1 .

Proposition 6.5. Fix a simplicial complex $\Delta$ of dimension $r$. The singularity dimension of $\Delta$ is the minimum $i$ such that $\Delta$ is $C M$ in codimension $r-i-1$.

Proof. If $\Delta$ has singularity dimension $s$, then $\Delta$ is CM of the appropriate dimension along every face of dimension $\geq s+1$ by definition and Reisner's criterion (if $1 \mathrm{k} F$ had too small dimension, then the link of any facet containing $F$ would give this fact away, by Remark 6.4); thus $\Delta$ is $\mathrm{CM}$ in codimension $r-s-1$. On the other hand, if $\Delta$ is $\mathrm{CM}$ in codimension $r-i-1$ then every face of dimension $i+1$ is nonsingular by definition.

Corollary 6.6. Fix a simplicial complex $\Delta$ of dimension $r$. The singularity dimension of $\Delta$ is less than $m$ if and only if $\Delta$ is $C M$ in codimension $r-m$.

This corollary and the next lemma form the bridge to general commutative algebra.

Lemma 6.7. A simplicial complex $\Delta$ is $C M$ of dimension $i$ along $F$ if and only if the localization $\mathbb{k}[\Delta]_{P_{F}}$ at the prime ideal $P_{F}=\left(x_{j}: j \notin F\right)$ of $F$ is a Cohen-Macaulay ring of Krull dimension $i+1$.

Proof. Localizing $\mathbb{k}[\Delta]$ by inverting the variables indexed by $F$ results in $\mathbb{k}[\operatorname{lk} F]\left[x_{i}^{ \pm 1}: i \in F\right]$. The lemma is straightforward from this and Reisner's criterion [9, Theorem 5.53].

Definition 6.8. A module $M$ over a noetherian ring is $C M$ of dimension $i$ locally at $\mathfrak{p}$ if the localization $M_{\mathfrak{p}}$ at the prime ideal $\mathfrak{p}$ is a Cohen-Macaulay module of Krull dimension $i$. If $\operatorname{dim} M=d$, then $M$ is $C M$ in codimension $c$ if $M$ is CM of dimension $c$ locally at every prime $\mathfrak{p}$ of dimension $d-c$ in the support of $M$, or if $c>d$ and $M$ is Cohen-Macaulay. 
Theorem 6.9. A simplicial complex $\Delta$ of dimension $r$ is $C M$ in codimension $c$ if and only if $\mathbb{k}[\Delta]$ is $C M$ in codimension $c$. In particular, the singularity dimension of $\Delta$ is less than $m$ if and only if $\mathbb{k}[\Delta]$ is $C M$ in codimension $r-m$.

Proof. Use Lemma 6.7 for the first sentence and add Corollary 6.6] for the second.

Remark 6.10. Definition 6.8 is related to, but different from, Serre's condition $S_{c}$. Both conditions can be interpreted as stipulating the Cohen-Macaulay condition near the prime ideals of certain large irreducible subvarieties, but $S_{c}$ requires a module to be Cohen-Macaulay near every prime whose local ring has depth at least $c$, whereas Definition 6.8 requires a module to be Cohen-Macaulay at every prime whose local ring has dimension at least $c$. These two conditions manifest differently in local cohomology: a module $M$ over a regular local ring with maximal ideal $\mathfrak{m}$ satisfies Serre's condition $S_{c}$ when its local cohomology $H_{\mathfrak{m}}^{i}(M)$ vanishes in cohomological degrees $i<c$, whereas $M$ is CM in codimension $c$ when the Matlis dual of its local cohomology satisfies $\operatorname{dim} H_{\mathfrak{m}}^{i}(M)^{\vee}<\operatorname{dim} M-c$ for $i<\operatorname{dim} M$ (apply local duality to Corollary 7.3 below). When $M$ is a ring, this means that the homology of its dualizing complex has dimension $<\operatorname{dim} M-c$ except at the end where it has $\operatorname{dimension} \operatorname{dim} M$. In short, Serre's condition bounds the cohomological degrees of the nonvanishing local cohomology modules, whereas CM in a fixed codimension bounds their Krull dimensions. See [10] for recent work on combinatorial implications of Serre's conditions on face rings.

\section{Modules Cohen-Macaulay in a fixed codimension}

With Theorem 6.9 in mind, Theorem 2.3 is a statement about the behavior, under quotients by generic linear forms, of certain graded rings that are Cohen-Macaulay in a fixed codimension. The end result of this section, Theorem [7.9, demonstrates the rather general nature of this statement: it holds for any finitely generated graded module over any finitely generated standard $\mathbb{Z}$-graded $\mathbb{k}$-algebra, where $\mathbb{k}$ is an infinite field.

Since the point of the developments of this section is to ignore certain modules of finite length, the main result is best proved in the language of sheaves on projective schemes, which we do in Theorem 7.7. In that formulation, it is especially easy to see how the developments here relate general Bertini-type theorems in modern algebraic geometry; see Remark 7.10.

We begin by rephrasing Definition 6.8 in geometric terms. Recall that the dimension of a point $\mathfrak{p}$ in a scheme is the supremum of the lengths $\ell$ of chains $\mathfrak{p}=\mathfrak{p}_{\ell}>\cdots>\mathfrak{p}_{1}>\mathfrak{p}_{0}$ where $\mathfrak{p}_{i}>\mathfrak{p}_{j}$ means that $\mathfrak{p}_{j}$ lies in the closure of $\mathfrak{p}_{i}$. We use the convention that a sheaf or module is zero if and only if its Krull dimension is negative.

Definition 7.1. A coherent sheaf $\mathcal{F}$ on a noetherian scheme with structure sheaf $\mathcal{O}$ is $C M$ of dimension $i$ locally at $\mathfrak{p}$ if the germ $\mathcal{F}_{\mathfrak{p}}$ is a Cohen-Macaulay $\mathcal{O}_{\mathfrak{p}}$-module of Krull dimension $i$. If $\operatorname{dim} \mathcal{F}=r$, then $\mathcal{F}$ is $C M$ in codimension $c$ if $\mathcal{F}$ is CM of dimension $c$ locally at every point $\mathfrak{p}$ of dimension $r-c$ in the support of $\mathcal{F}$, or if $c>r$ and $\mathcal{F}$ is Cohen-Macaulay.

Proposition 7.2. A coherent sheaf $\mathcal{F}$ of dimension $r$ on a regular scheme $X$ of dimension $\delta$ is $C M$ in codimension $c$ if and only if $\operatorname{dim} \mathcal{E} x t_{X}^{i}\left(\mathcal{F}, \mathcal{O}_{X}\right)<r-c$ for $i>\delta-r$. 
Proof. The sheaf $\mathcal{E x t}_{X}^{i}\left(\mathcal{F}, \mathcal{O}_{X}\right)$ has dimension $<r-c$ if and only if its germ at every point $\mathfrak{p}$ of dimension $r-c$ is zero. The germ in question is $\mathcal{E x t}_{X}^{i}\left(\mathcal{F}, \mathcal{O}_{X}\right)_{\mathfrak{p}}=\operatorname{Ext}_{\mathcal{O}_{X, \mathfrak{p}}}^{i}\left(\mathcal{F}_{\mathfrak{p}}, \mathcal{O}_{X, \mathfrak{p}}\right)$ because $\mathcal{F}$ is coherent and $X$ is noetherian. At any point $\mathfrak{p}$ in the support of $\mathcal{F}$, the germ $\mathcal{F}_{\mathfrak{p}}$ at $\mathfrak{p}$ has dimension at most $r-\operatorname{dim} \mathfrak{p}$ and depth equal to $\delta-\operatorname{dim} \mathfrak{p}-\max \left\{i: \operatorname{Ext}_{\mathcal{O}_{X, \mathfrak{p}}}^{i}\left(\mathcal{F}_{\mathfrak{p}}, \mathcal{O}_{X, \mathfrak{p}}\right) \neq 0\right\}$, the latter by [1, Exercise 3.1.24]. The dimension and depth equal $c$ for all points $\mathfrak{p}$ of dimension $r-c$ if and only if the maximum is $\delta-r$ for all such $\mathfrak{p}$, and the first sentence of the proof implies that this is equivalent to the desired dimension condition on $\operatorname{Ext}_{X}^{i}\left(\mathcal{F}, \mathcal{O}_{X}\right)$.

The following algebraic version is immediate from the affine case of Proposition 7.2 .

Corollary 7.3. A finitely generated module $M$ of dimension d over $S=\mathbb{k}\left[x_{1}, \ldots, x_{n}\right]$ is $C M$ in codimension $c$ if and only if $\operatorname{dim}_{\operatorname{Ext}_{S}^{i}}(M, S)<d-c$ for $i>n-d$.

Applying the above corollary to the second sentence of Theorem 6.9 yields the following important consequence, noting that $c=r-m \Leftrightarrow d-c=m+1$ when $r=d-1$.

Corollary 7.4. A simplicial complex $\Delta$ of dimension $r=d-1$ has singularity dimension less than $m$ if and only if $\operatorname{dim}_{\operatorname{Ext}_{S}^{i}}(\mathbb{k}[\Delta], S) \leq m$ for $i>n-d$.

Remark 7.5. Since local cohomology and Ext into $S$ are Matlis dual by local duality, and since the Krull dimension of a module $M$ equals the order to which $\lambda=1$ is a pole of the $\mathbb{Z}$-graded Hilbert series $F(M, \lambda)$ of $M$, Corollary 7.4 is equivalent to Corollary 3.2 ,

Another consequence of Proposition 7.2 is a characterization of finite local cohomology.

Corollary 7.6. A finitely generated standard $\mathbb{Z}$-graded $S$-module $M$ of dimension $d$ has finite local cohomology if and only if $M$ is CM in codimension $d-1$.

Proof. The module $\operatorname{Ext}_{S}^{i}(M, S)$ is the graded Matlis dual of $H_{\mathfrak{m}}^{n-i}(M)$ by local duality. Thus $M$ has finite local cohomology if and only if $\operatorname{dim}_{\operatorname{Ext}_{S}^{i}}(M, S) \leq 0$ for $i>n-d$.

Next is the main result of this section, a Bertini-type theorem for CM in codimension $c$.

Theorem 7.7. Fix a coherent sheaf $\mathcal{F}$ on $\mathbb{P}^{n}$ and a linear form $\theta$ on $\mathbb{P}^{n}$ vanishing on a hyperplane $H$. Assume that $\theta$ is nonzero at every associated point of $\bigoplus_{i} \mathcal{E x t}_{\mathbb{P} n}^{i}\left(\mathcal{F}, \mathcal{O}_{\mathbb{P} n}\right)$. For $c<\operatorname{dim} \mathcal{F}$, the sheaf $\mathcal{F}$ is $C M$ in codimension $c$ if and only if its restriction $\left.\mathcal{F}\right|_{H}$ is. If $\mathcal{F}$ is Cohen-Macaulay (this is the case of $c \geq \operatorname{dim} \mathcal{F}$ ), its restriction $\left.\mathcal{F}\right|_{H}$ is also Cohen-Macaulay.

Proof. As the final sentence is well-known for Cohen-Macaulay sheaves, we fix $c<\operatorname{dim} \mathcal{F}$. Let $\mathcal{E}$. be a resolution of $\mathcal{F}$ by locally free sheaves, and write $\mathcal{E}^{\bullet}$ for the dual complex of sheaves. Thus the cohomology of $\mathcal{E}^{\bullet}$ is $\mathcal{E x t}_{\mathbb{P}^{n}}\left(\mathcal{F}, \mathcal{O}_{\mathbb{P}^{n}}\right)$. The restriction $\left.\mathcal{E}^{\bullet}\right|_{H}$ to $H$ is the cokernel of the sheaf homomorphism $\mathcal{E}^{\bullet}(-1) \rightarrow \mathcal{E}^{\bullet}$ induced by $\theta$. Its cohomology is $\mathcal{E} x t_{H}^{\bullet}\left(\left.\mathcal{F}\right|_{H}, \mathcal{O}_{H}\right)$; this is by the genericity hypothesis on $\theta$, which also guarantees that the cohomology sheaves of $\left.\mathcal{E}^{\bullet}\right|_{H}$ are obtained by restricting those of $\mathcal{E}^{\bullet}$ to $H$. The dimensions of the cohomology sheaves decrease by precisely 1 (recall our negative dimension convention) by genericity again and the Hauptidealsatz; geometrically: $H$ is transverse to every component in the support of each $\mathcal{E} x t$ sheaf. Consequently, $\operatorname{dim} \mathcal{E} x t_{\mathbb{P}^{n}}^{\bullet}\left(\mathcal{F}, \mathcal{O}_{\mathbb{P}^{n}}\right)<\operatorname{dim} \mathcal{F}-c \Leftrightarrow \operatorname{dim} \mathcal{E} x t_{H}^{\bullet}\left(\left.\mathcal{F}\right|_{H}, \mathcal{O}_{H}\right)<\operatorname{dim} \mathcal{F}_{H}-c$, where the " $\Leftarrow$ " direction requires the hypothesis $\operatorname{dim} \mathcal{F}-c>0$. Now apply Proposition 7.2 to $\mathcal{E} x t_{\mathbb{P}^{n}}^{\bullet}\left(\mathcal{F}, \mathcal{O}_{\mathbb{P}^{n}}\right)$ and $\mathcal{E} x t_{H}^{\bullet}\left(\left.\mathcal{F}\right|_{H}, \mathcal{O}_{H}\right)$ to complete the proof. 
Proposition 7.8. Fix a graded $S$-module $M$ with associated sheaf $\mathcal{F}$ on projective space $\mathbb{P}^{n-1}$. If $c \leq \operatorname{dim} \mathcal{F}$, then $M$ is $C M$ in codimension $c$ if and only if $\mathcal{F}$ is $C M$ in codimension $c$.

Proof. By functoriality, $\mathcal{E} x t_{X}^{i}\left(\mathcal{F}, \mathcal{O}_{X}\right)$ is the sheaf associated to $\operatorname{Ext}_{S}^{i}(M, S)$, where $X=\mathbb{P}^{n-1}$, hence the the result is a straightforward consequence of Proposition 7.2 and Corollary 7.3 .

The following algebraic version of Theorem 7.7 is slightly less clean for $c \approx \operatorname{dim} M$, owing to the possibility of the maximal ideal $\mathfrak{m}$ as an associated prime of $M$ and its Ext modules.

Theorem 7.9. Fix a graded $S$-module $M$ and a linear form $\theta$ not in any associated prime $\neq \mathfrak{m}$ of $\bigoplus_{i} \operatorname{Ext}_{S}^{i}(M, S)$. If $c<\operatorname{dim} M-1$ then $M / \theta M$ is $C M$ in codimension $c$ if and only if $M$ is. If $M$ has finite local cohomology or is Cohen-Macaulay then so is $M / \theta M$, accordingly.

Proof. If $c<\operatorname{dim} M-1$, then the result follows from Theorem 7.7 and Proposition 7.8. If $c \geq \operatorname{dim} M-1$ and $M$ is $\mathrm{CM}$ in codimension $c$, then (by Corollary 7.6) the sheaf on $\mathbb{P}^{n-1}$ associated to $M$ is Cohen-Macaulay, and so the same is true of the sheaf associated to $M / \theta M$ by Theorem [7.7, but $M / \theta M$ might itself have (nonzero) finite local cohomology.

Finally, it remains to see that Theorem 7.9 does indeed directly generalize Theorem 2.3 ,

Proof of Theorem 2.3. Let $\Delta$ have dimension $r=d-1$, so $\mathbb{k}[\Delta]$ has dimension $d$. The quotient $\mathbb{k}[\Delta] /\left(\theta_{1}, \ldots, \theta_{m}\right)$ modulo a sequence of $m$ generic linear forms has finite local cohomology if and only if it is CM in codimension $(d-m)-1=r-m$, by Corollary 7.6. This occurs if and only if $\mathbb{k}[\Delta]$ itself is CM in codimension $r-m$, by Theorem 7.9 , The desired result follows from the characterization of singularity dimension in Theorem 6.9.

Remark 7.10. The first result in the general direction of Theorems 7.7 and 7.9 was proved by Flenner for subschemes of spectra of local rings [4, Satz 3.3]. It is a special case of Spreafico's very general Bertini-type theorem for local geometric conditions satisfying certain natural axioms [14, Corollary 4.3]; for the purposes here, the reader can check that $\mathrm{CM}$ in codimension $c$ is a valid choice for Spreafico's "property $\mathbb{P}$ ". However, Flenner and Spreafico proved their results for subschemes, not coherent sheaves, and the "if" direction of Theorems 7.7 and 7.9 does not fall under Spreafico's framework: one cannot conclude that a variety is (for instance) smooth by knowing that a generic hyperplane section is smooth. In contrast, the constrained version of the Cohen-Macaulay condition furnished by Definition 7.1 lifts from hyperplane sections because a hyperplane in projective space intersects every subvariety of positive dimension and - generically, at least - reduces its dimension by 1 , the operative subvariety being the locus where a sheaf fails to be CM in codimension $c$. The failure of hyperplanes to meet subschemes of dimension 0 accounts for the failure of the "if" direction when the codimension $c$ is large. 


\section{Squarefree modules modulo systems of parameters}

In Section 5, we derived the simplicial Theorem 2.3 from the simplicial calculations of Hilbert series of local cohomology modules that constitute Theorem 5.3. Having taken the former to its natural generality in Sections 6 and 7, we now generalize the latter in Theorem 8.5, where $H_{F}^{\ell+m}(M)=H_{F}^{\ell+m-1}(\Delta)$ when $M=\mathbb{k}[\Delta]$ by Theorem 3.1. It is possible to imagine that generalizations to arbitrary $\mathbb{N}^{n}$-graded modules could exist, but we limit ourselves here to the class of squarefree modules, introduced by Yanagawa [19].

Definition 8.1. A $\mathbb{Z}^{n}$-graded module over $S=\mathbb{k}\left[x_{1}, \ldots, x_{n}\right]$ is squarefree if its generators and relations all lie in squarefree degrees, meaning those in $\{0,1\}^{n} \subset \mathbb{Z}^{n}$.

The point of squarefree modules is that, like face rings $\mathbb{k}[\Delta]$, all of the information can be recovered easily from the finite-dimensional vector subspace in squarefree degrees.

One of the fundamental conclusions of Section 5 is that the entirety of the interesting combinatorial information encoded by the local cohomology of $\mathbb{k}[\Delta]$ is distilled into the finite length modules in $\mathbb{Z}$-graded degrees zero and above that remain after applying Theorem 5.3 enough times. Other types of reductions to vector spaces of finite-dimension are known for the local cohomology of $\mathbb{k}[\Delta]$, and indeed of any squarefree module $M$ : one way to phrase it is that the $\mathbb{Z}^{n}$-graded Matlis dual $H_{\mathfrak{m}}^{i}(M)^{\vee}=\operatorname{Ext}_{S}^{n-i}\left(M, \omega_{S}\right)$ is a squarefree module [19, Theorem 2.6], where the canonical module $\omega_{S}$ is the rank 1 free module generated in $\mathbb{Z}^{n}$-graded degree $(1, \ldots, 1)$. (An analogous statement holds for the local cohomology of arbitrary $\mathbb{N}^{n}$-graded modules: the Čech hull [7, Definition 2.7] recovers the local cohomology from an a priori bounded set of degrees; see the proof of [8, Lemma 3.10].)

In fact, the squarefreeness of these Ext modules is the key to Theorem 8.5, via the general enumerative statement about arbitrary squarefree modules in Proposition 8.3, First, we need a lemma, whose proof relies on a key feature (the squarefree filtration) of squarefree modules.

Lemma 8.2. If $M$ is a squarefree $S$-module and $R=S / \Theta S$ for a sequence $\Theta=\theta_{1}, \ldots, \theta_{m}$ of generic linear forms, then $\operatorname{Tor}_{t}^{S}(M, R)_{i}=0$ whenever $t>0$ and $i>m$.

Proof. For each squarefree vector $F$, set $\mathbf{x}^{F}=\prod_{j \in F} x_{j}$ and write $\mathbb{k} F$ for the $\mathbb{Z}^{n}$-graded $S$ module $\mathbf{x}^{F} \mathbb{k}\left[x_{j}: j \in F\right]=\mathbf{x}^{F} S /\left(x_{j}: j \notin F\right)$ generated in degree $F$. As a standard $\mathbb{Z}$-graded module, $\mathbb{k} F$ is a polynomial ring in $|F|$ many variables generated in degree $|F|$.

The squarefree module $M$ has a filtration

$$
0=M_{0} \subset M_{1} \subset \cdots \subset M_{k}=M
$$

such that each quotient $M_{j} / M_{j-1}$ is isomorphic, as a $\mathbb{Z}^{n}$-graded module, to $\mathbb{k} F$ for some squarefree vector $F[19$, Proposition 2.5]. If the filtration has length 1 , and so $M=\mathbb{k} F$, then the module $\operatorname{Tor}_{t}^{S}(\mathbb{k} F, R)$ vanishes for $t>0$ whenever $|F| \geq m$. This is because $\Theta$ is a regular sequence on $\mathbb{k} F$ in that case. When $|F|<m$, on the other hand, $\operatorname{Tor}_{t}^{S}(\mathbb{k} F, R) \cong$ $\operatorname{Tor}_{t}^{S^{\prime}}(\mathbb{k}(-|F|), R)$, where $S^{\prime}=S /\left(\theta_{1}, \ldots, \theta_{|F|}\right)$ and $\mathbb{k}(-|F|)=\mathbb{k} F \otimes_{S} S^{\prime}$. Thus the Tor in question is a direct sum of $\left(\begin{array}{c}m-|F| \\ t\end{array}\right)$ many copies of the residue field $\mathbb{k}$ in $\mathbb{Z}$-graded degree $|F|+t$. The desired result holds for $M=\mathbb{k} F$ since $\left(\begin{array}{c}m-|F| \\ t\end{array}\right)=0$ as soon as $t>m-|F|$. 
The general case follows by induction on the length of the filtration (8) by tensoring the exact sequence $0 \rightarrow M_{j-1} \rightarrow M_{j} \rightarrow M_{j} / M_{j-1} \rightarrow 0$ with the Koszul complex on $\Theta$.

Proposition 8.3. Fix a squarefree module $E$ and a sequence $\Theta=\theta_{1}, \ldots, \theta_{m}$ of generic linear forms. For all $i \geq 0$, the vector space dimension of the $i$-th standard $\mathbb{Z}$-graded piece of $E$ is

$$
\operatorname{dim}_{\mathbb{k}} E_{i}=\sum_{F \in\{0,1\}^{n}}\left(\begin{array}{c}
i-1 \\
|F|-1
\end{array}\right) \cdot \operatorname{dim}_{\mathbb{k}} E_{F},
$$

where $E_{F}$ is the piece of $E$ in $\mathbb{Z}^{n}$-graded degree $F$. In contrast, for all $i>m$,

$$
\operatorname{dim}_{\mathbb{k}}(E / \Theta E)_{i}=\sum_{F \in\{0,1\}^{n}}\left(\begin{array}{c}
i-m-1 \\
|F|-m-1
\end{array}\right) \cdot \operatorname{dim}_{\mathbb{k}} E_{F} .
$$

Proof. The formula for $\operatorname{dim}_{\mathbb{k}} E_{i}$ simply expresses the fact that $E$ and its associated graded module for the squarefree filtration (8) have the same Hilbert function, which is the sum of the Hilbert functions of the associated graded modules $\mathbb{k} F$; the binomial coefficient counts the monomials of degree $i-|F|$ in (at most) $|F|$ many variables.

The binomial coefficient in the formula for $\operatorname{dim}_{\mathbb{k}}(E / \Theta E)_{i}$ counts the monomials of degree $i-|F|$ in $|F|-m$ variables, which is the dimension of the $i$-th $\mathbb{Z}$-graded piece of $\mathbb{k} F / \Theta \mathbb{k} F$. It therefore suffices to show that the Hilbert functions of $E / \Theta E$ and gr $E / \Theta$ gr $E$ agree in degrees $>m$. To see this, tensor the short exact sequence $0 \rightarrow E_{j-1} \rightarrow E_{j} \rightarrow E_{j} / E_{j-1} \rightarrow 0$ with $S / \Theta$ and note that it remains exact in $\mathbb{Z}$-graded degrees $>m$ by Lemma 8.2 .

For the next result, assume that a system $\Theta=\theta_{1}, \ldots, \theta_{m}$ of generic linear forms has been fixed, and set $R=S / \Theta S$. The standard $\mathbb{Z}$-graded canonical module of $S$ is $\omega_{S}=S(-n)$, whereas the $\mathbb{Z}$-graded canonical module of $R$ is $\omega_{R}=R(-(n-m))=R \otimes_{S} \omega_{S}(m)$.

Proposition 8.4. For any standard $\mathbb{Z}$-graded $S$-module $M$, the sheaves on projective space determined by $R \otimes_{S} \operatorname{Ext}_{S}^{\ell}\left(M, \omega_{S}\right)(m)$ and $\operatorname{Ext}_{R}^{\ell}\left(R \otimes_{S} M, \omega_{R}\right)$ are isomorphic; that is, the modules themselves are isomorphic up to a kernel and cokernel supported at the maximal ideal.

Proof. Reasoning as in the proofs of Theorem 7.7 and Proposition 7.8 demonstrates that $R \otimes_{S} \operatorname{Ext}_{S}^{\ell}(M, S)$ and $\operatorname{Ext}_{R}^{\ell}\left(R \otimes_{S} M, R\right)$ determine isomorphic sheaves. Beyond that, all that remains is fiddling with the $\mathbb{Z}$-graded degree shifts.

Theorem 8.5. Fix a squarefree $S$-module $M$ and a sequence $\Theta=\theta_{1}, \ldots, \theta_{m}$ of generic linear forms. If $H_{F}^{j}(M):=H_{\mathfrak{m}}^{j}(M)_{-F}$ is the $\mathbb{Z}^{n}$-graded piece of the $j$-th local cohomology of $M$ in the negative of the squarefree degree $F$, then for all $i \gg 0$,

$$
\operatorname{dim}_{\mathfrak{k}} H_{\mathfrak{m}}^{\ell}(M / \Theta M)_{-i}=\sum_{F}\left(\begin{array}{c}
i-1 \\
|F|-m-1
\end{array}\right) \cdot \operatorname{dim}_{\mathfrak{k}} H_{F}^{\ell+m}(M) .
$$


Proof. Using $\omega_{S}=x_{1} \cdots x_{n} S=S(-1, \ldots,-1)$ and an asterisk for vector space duals, $H_{\mathfrak{m}}^{\ell+m}(M)_{-\alpha} \cong \operatorname{Ext}_{S}^{n-m-\ell}\left(M, \omega_{S}\right)_{\alpha}^{*}$ for all $\alpha \in \mathbb{Z}^{n}$ by $\mathbb{Z}^{n}$-graded local duality [7, Corollary 6.1]. Thus $H_{F}^{j}(M) \cong \operatorname{Ext}_{S}^{n-j}\left(M, \omega_{S}\right)_{F}^{*}$ for all cohomological degrees $j$ and all squarefree vectors $F$, and so Proposition 8.3 implies that the right-hand side of the desired formula is the dimension of the $\mathbb{Z}$-graded piece $R \otimes \operatorname{Ext}_{S}^{n-m-\ell}\left(M, \omega_{S}\right)_{i+m}$. The result follows from Proposition 8.4, given that $H_{\mathfrak{m}}^{\ell}(M / \Theta M)_{-i} \cong \operatorname{Ext}_{R}^{n-m-\ell}\left(R \otimes M, \omega_{R}\right)_{i}^{*}$ by $\mathbb{Z}$-graded local duality [1, Theorem 3.6.19].

The special case of Theorem 8.5 in which $M=\mathbb{k}[\Delta]$ is slightly weaker than Theorem 5.3, because of the condition $i \gg 0$, but we believe this to be an artifact of the proof.

Conjecture 8.6. The formula for $\operatorname{dim}_{\mathbb{k}} H_{\mathfrak{m}}^{\ell}(M / \Theta M)_{-i}$ in Theorem 8.5 holds for all $i>0$.

Remark 8.7. The conjecture easily reduces to a more precise version of Proposition 8.4; we need $R \otimes_{S} \operatorname{Ext}_{S}^{\ell}\left(M, \omega_{S}\right)(m)$ and $\operatorname{Ext}_{R}^{\ell}\left(R \otimes_{S} M, \omega_{R}\right)$ to be isomorphic in all positive $\mathbb{Z}$-graded degrees when $M$ is a squarefree module. This probably follows from the full strength of Lemma 8.2 (only the case $t=1$ was used in the proof of Proposition 8.3) and a comparison between $\operatorname{Ext}_{R}^{\ell}\left(R \otimes_{S} M, \omega_{R}\right)$ and the cohomology of the total complex of $\operatorname{Hom}_{S}\left(K_{\bullet} \otimes F_{\bullet}, \omega_{S}\right)(m)$, where $K_{\bullet}=K_{\bullet}(\Theta)$ is the Koszul complex of $\theta_{1}, \ldots, \theta_{m}$ and $F_{\bullet}$ is a resolution of $M$ by graded free $S$-modules. However, the derivation of Theorem 2.3 in Section 5 only requires the weakened version of Theorem 5.3 that is an immediate consequence

of Theorem 8.5 as stated. Therefore, since the conjecture is not central to our conclusions regarding finite local cohomology, we leave it open.

\section{References}

[1] W. Bruns and J. Herzog, Cohen-Macaulay rings, Cambridge Studies in Advanced Mathematics, vol. 39, Cambridge University Press, Cambridge, 1993.

[2] N.T. Coung, P. Schenzel, and N.V. Trung, Verallgemeinerte Cohen-Macaulay-Moduln, Math. Nachr. 85 (1978), 57-73.

[3] D. Eisenbud, Commutative Algebra with a View Toward Algebraic Geometry, SpringerVerlag, New York, 1995.

[4] H. Flenner, Die Sätze von Bertini für lokale Ringe, Math. Ann. 229 (1977), no. 2, 97-111.

[5] S. Goto and Y. Takayama, Stanley-Reisner ideals whose powers have finite length cohomologies, Proc. Amer. Math. Soc. 135 (2007), 2355-2364.

[6] H.-G. Gräbe, The canonical module of a Stanley-Reisner ring, J. Algebra 86 (1984), $272-281$.

[7] E. Miller, The Alexander duality functors and local duality with monomial support, J. Algebra 231 (2000), 180-234. 
[8] E. Miller, Topological Cohen-Macaulay criteria for monomial ideals, in Combinatorial aspects of commutative algebra (Viviana Ene and Ezra Miller, eds.), Contemporary Mathematics, Vol. 502, American Mathematical Society, Providence, RI, 2009, 137156. arXiv:math.AC/0809.1458

[9] E. Miller and B. Sturmfels, Combinatorial Commutative Algebra, Graduate Texts in Mathematics, vol. 227, Springer-Verlag, New York, 2005.

[10] S. Murai and N. Terai, h-vectors of simplicial complexes with Serre's conditions, preprint. arXiv:math.AC/0912.1089

[11] G. Reisner, Cohen-Macaulay quotients of polynomial rings, Adv. in Math. 21 (1976), 30-49.

[12] U. Schäfer and P. Schenzel, Dualizing complexes of affine semigroup rings, Trans. Amer. Math. Soc. 322 (1990), no. 2, 561-582.

[13] P. Schenzel, On the number of faces of simplicial complexes and the purity of Frobenius, Math. Z. 178 (1981), 125-142.

[14] M. L. Spreafico, Axiomatic theory for transversality and Bertini type theorems, Arch. Math. (Basel) 70 (1998), no. 5, 407-424.

[15] R. Stanley, Cohen-Macaulay complexes, in Higher Combinatorics (M. Aigner ed.), Reidel, Dordrecht and Boston, 1977, 51-62.

[16] R. Stanley, Combinatorics and Commutative Algebra, Birkhäuser, 1996.

[17] J. Stückrad and W. Vogel, Buchsbaum Rings and Applications, Springer-Verlag, Berlin, 1986.

[18] N.V. Trung, Toward a theory of generalized Cohen-Macaulay modules, Nagoya Math. J. 102 (1986), 1-49.

[19] K. Yanagawa, Alexander duality for Stanley-Reisner rings and squarefree $\mathbb{N}^{n}$-graded modules, J. Algebra 225 (2000), no. 2, 630-645.

[20] Y. Takayama, Combinatorial characterizations of generalized Cohen-Macaulay ideals, Bull. Math. Soc. Sci. Math. Roumanie 48(96) (2005), 327-344. 